Monika Linne, Ines Drefs, Nora Dörrenbächer, Pascal Siegers, Mathias Bug

\title{
3.2 GO FAIR und GO CHANGE: Chancen für das deutsche Wissenschaftssystem
}

\begin{abstract}
Der vorliegende Beitrag beschäftigt sich mit der GO FAIR-Initiative und deren angestrebten Auswirkungen auf das deutsche Wissenschaftssystem. Die Initiative hat das Ziel, die Auffindbarkeit, Zugänglichkeit, Interoperabilität und Wiederverwendbarkeit von Forschungsdaten zu verbessern. Ihr offener und bottom-upgestützter Ansatz zielt auf die Einbindung aller Forschungsbereiche und EU-Mitgliedsstaaten ab und soll Forschungsdaten zukünftig länder- und disziplinübergreifend nachnutzbar machen. Da Forschungsfelder immer häufiger interdisziplinärer Natur sind, ist dies für den Erkenntnisfortschritt in der datenbasierten und immer stärker digitalisierten Forschung - letztendlich für das gesamte Wissenschaftssystem - ein bedeutender Mehrwert.

Speziell zur thematischen Sensibilisierung der im Wissenschaftssystem Tätigen wurde das Handlungsfeld GO CHANGE eingerichtet. Es soll dabei unterstützen, einen verantwortungsvollen und professionellen Umgang mit FAIRen Forschungsdaten zu kultivieren. Im Folgenden wird zunächst ein Überblick über die GO FAIR-Initiative und deren Struktur gegeben. Von besonderer Bedeutung sind hierbei die Implementierungsnetzwerke (IN), welche sich im Handlungsfeld GO CHANGE gegründet haben. Exemplarisch werden anhand des Implementierungsnetzwerkes für Sozial-, Verhaltens- und Wirtschaftswissenschaften (Eco-SocIN) die Spannbreite, Nutzungscommunity und Beteiligungsmöglichkeiten am Datenkulturwandel aufgezeigt, wie er auch für sensible Daten anzustreben ist. Insgesamt soll dieser Artikel die Relevanz von GO CHANGE und die damit verbundenen Bemühungen und Ziele herausstellen und die positiven Auswirkungen auf das deutsche Wissenschaftssystem verdeutlichen.
\end{abstract}

\section{Einleitung}

Digitalisierungsprozesse in der Wissenschaft eröffnen grundlegend neue Möglichkeiten für einen interdisziplinären Forschungsprozess und Erkenntnisgewinn, was sich insbesondere auf Forschungsmethoden und Forschungsdaten niederschlägt. Berechtigterweise werden solche Prozesse deshalb von Seiten wissenschaftspolitischer Akteurinnen und Akteuren unterstützt und gezielt vorangetrieben. Problematisch ist jedoch, dass die Etablierung eines hierzu notwendigen Forschungsdatenmanagements (FDM) disziplinübergreifend in Deutschland trotz einiger guter Beispiele bislang weitestgehend durch zeitlich begrenzte Initiativen geprägt ist. Diese 
Initiativen sind größtenteils nur gering koordiniert und werden auf Projektbasis durch Drittmittel finanziert. In diesem Sinne fehlt aus Sicht des Rats für Informationsinfrastrukturen (RfII) ${ }^{1}$ eine nachhaltige Grundversorgung der Forschenden mit niedrigschwelligen und dauerhaften FDM-Services für das Forschungsdatenmanagement und es besteht Handlungsbedarf in zahlreichen Feldern. ${ }^{2}$ Forschende benötigen zuverlässige Unterstützung und Beratung zur nachhaltigen Datendokumentation (Metadaten), Datenorganisation (Dateiformate, Datenaustausch), Datenspeicherung und -archivierung, rechtliche Rahmenbedingungen (Urheberrecht, Datenschutz, Lizenzierung) und Möglichkeiten zur Datenpublikation (Repositorium, Datenzentren, Persistente Identifier).

Zusätzlich zu den FDM-Services und notwendigen Tools mangelt es parallel an einem Mentalitätswandel hin zu einer größeren Akzeptanz des Forschungsdatenmanagements als wissenschaftliche Leistung seitens der Forschenden und deren Communities. Das enorme (interdisziplinäre) Forschungspotenzial, welches aus einem strukturierten FDM resultiert, wurde von Forschenden, aber auch von einigen wissenschaftlichen Einrichtungen noch nicht erkannt. So liegt es den meisten Forschenden leider immer noch fern, eine etwaige Archivierung oder gar Veröffentlichung ihrer Forschungsdaten von Beginn an in ihre Forschungsprozesse zu integrieren. Dieser Umstand ist nicht zuletzt auch auf einen befürchteten Mehraufwand und die Sorge vor Kontrollverlust zurückzuführen. ${ }^{3}$ An dieser Stelle setzt GO CHANGE an. Hierbei handelt es sich um ein Handlungsfeld der GO FAIR-Initiative, die sich als interdisziplinäres Netzwerk versteht, „bestehend aus Personen und Organisationen, die sich in unterschiedlichen Projekten auf der ganzen Welt dafür engagieren, Forschungsobjekte auffindbar, zugänglich, interoperabel und wiederverwendbar zu machen". ${ }^{4}$

Zentrale Forderung der GO FAIR-Initiative ist, dass Forschungsdaten den so genannten FAIR-Prinzipien entsprechen und auffindbar (Findable), zugänglich (Accessible), interoperabel (Interoperable) sowie wiederverwendbar (Re-usable) sein sollen. ${ }^{5}$ Diese Forderung basiert auf einem breiten wissenschaftspolitischen Konsens. So verlangen beispielsweise die Förderkriterien zum Aufbau der Nationalen Forschungsdateninfrastruktur (NFDI) ${ }^{6}$ in Deutschland eine „strategy for data use, access, findability and reusability in accordance with the FAIR principles“7 von den

\footnotetext{
1 S. http://www.rfii.de. Letztes Abrufdatum der Internet-Dokumente ist der 15.11.2020.

2 Vgl. RfII 2016, 37.

3 Vgl. Zenk-Möltgen et al. 2018; Beitrag von Oßwald, Kap. 3.5 in diesem Praxishandbuch.

4 Drefs et al. 2018, 638.

5 Vgl. Wilkinson et al. 2016.

6 S. https://www.dfg.de/foerderung/programme/nfdi/.

7 DFG 2019, 2. S. a. Beitrag von Neuroth und Oevel, Kap. „Aktuelle Entwicklung und Herausforderungen im Forschungsdatenmanagement in Deutschland“ in diesem Praxishandbuch.
} 
sich bewerbenden Konsortien. Auf europäischer Ebene gelten die FAIR-Prinzipien als wichtige Grundsätze für den Aufbau der European Open Science Cloud (EOSC). ${ }^{8}$ Zur Erarbeitung klarer Umsetzungsempfehlungen hat das Executive Board der EOSC unlängst eine spezialisierte Arbeitsgruppe eingesetzt, die „FAIR working group“.9 Auf globaler Ebene wäre letztlich sogar ein umfassendes „Internet of FAIR Data and Services (IFDS)“ denkbar bzw. anzustreben. ${ }^{10}$ Während digitale Wissensinfrastrukturen wie die NFDI, die EOSC oder das IFDS noch im Entwicklungsstadium stehen und somit relativ abstrakt sind, stellen sich in der Praxis schon ganz konkrete Fragen: Wie kann es gelingen, die FAIR-Prinzipien dort zu etablieren, wo sie letztendlich umgesetzt werden müssen, nämlich dort, wo Daten als Grundlage des Forschungsprozesses entstehen: in den Laboren und Büros der Forschenden? Wie kann FAIRes FDM fester Bestandteil guter wissenschaftlicher Praxis werden? Welche Anreize müssen geschaffen werden, damit Forschende eine extrinsische oder sogar intrinsische Motivation zum Data Sharing entwickeln? Hierfür ist ein Kulturwandel nötig, zumal es in der herrschenden Wissenschaftskultur einiges an Vorbehalten gegenüber dem Teilen von Forschungsdaten gibt. Einen solchen Mentalitätswandel zu befördern, hat sich die GO FAIR-Initiative mit dem Handlungsfeld GO CHANGE zum Ziel gesetzt, dessen Inhalte und Ziele in diesem Beitrag näher beschrieben werden sollen.

\section{Die GO FAIR-Initiative}

Die GO FAIR-Initiative verfolgt das Ziel, die Akzeptanz der FAIR-Prinzipien zu steigern und auf ihre breite Anwendung hinzuwirken. Den Startschuss hierfür bildete Ende 2017 eine gemeinsame Vereinbarung des Bundesministeriums für Bildung und Forschung (BMBF) und der Wissenschaftsministerien der Niederlande und Frankreich, die GO FAIR-Initiative durch die Einrichtung eines sogenannten „GO FAIR International Support and Coordination Office“ (im Folgenden „GO FAIR-Büro“ genannt) zu unterstützen. Die Idee, die dahinterstand, war, Vorreitern auf dem Gebiet des FDM eine niedrigschwellige Möglichkeit zu bieten, gemeinsam an der Umsetzung der FAIR-Prinzipien zu arbeiten - auch jenseits von großen Forschungsprojekten und etablierten Informationsinfrastrukturen. Auf diese Weise sollen in der aktuellen hochdynamischen Entwicklungsphase innerhalb des FDM redundante Entwicklungen vermieden werden und stattdessen bereits entwickelte Lösungen breite Anwendung finden.

8 Vgl. EOSC Declaration 2017; Beitrag von Streit und van Wezel, Kap. 1.2 in diesem Praxishandbuch. 9 Vgl. EOSCsecretariat 2019. 10 Vgl. Ayris et al. 2016, 12. 
Das von den drei Wissenschaftsministerien geförderte Büro hat demnach die Aufgabe, ein länder- und disziplinenübergreifendes Netzwerk aus Personen und Organisationen aufzubauen, die sich für die Auffindbarkeit, Zugänglichkeit, Interoperabilität und Wiederverwendbarkeit von Forschungsdaten engagieren - sei es in Bezug auf technische Lösungen, Aus- und Weiterbildungskonzepte oder eben den notwendigen kulturellen Wandel unter Forschenden. Unter dem Dach der GO FAIR-Initiative können sich die interessierten Akteurinnen und Akteure in so genannten „Implementierungsnetzwerken“ (IN) zusammenfinden, um auf bestimmte Herausforderungen bei der Umsetzung der FAIR-Prinzipien zu fokussieren und diese gemeinsam anzugehen. Seit Anfang 2018 ist das GO FAIR-Büro an den Standorten Hamburg (Deutschland), Leiden (Niederlande) und Paris (Frankreich) aktiv. Am Hamburger Standort ist es an der ZBW - Leibniz-Informationszentrum Wirtschaft ${ }^{11}$ angesiedelt. Hier koordinieren und unterstützen wissenschaftliche Referentinnen und Referenten die GO FAIR-Initiative auf vielfältige Weise. Sie identifizieren Synergiepotenziale zwischen bestehenden und zukünftigen GO FAIR-Akteurinnen und -Akteuren und bieten zu diesem Zweck beispielsweise Vernetzungsmöglichkeiten auf organisierten Workshops und Jahrestreffen. Außerdem sorgen die Referentinnen und Referenten dafür, dass Standards und Best Practices, die von sogenannten GO FAIR-Implementierungsnetzwerken entwickelt werden, möglichst weite Verbreitung finden, indem sie Projektergebnisse verständlich und zielgruppengerecht aufarbeiten. Im folgenden Kapitel soll dargestellt werden, wie FDM-Akteurinnen und -Akteure einem IN beitreten oder selber die Initiative zur Gründung eines solchen ergreifen können.

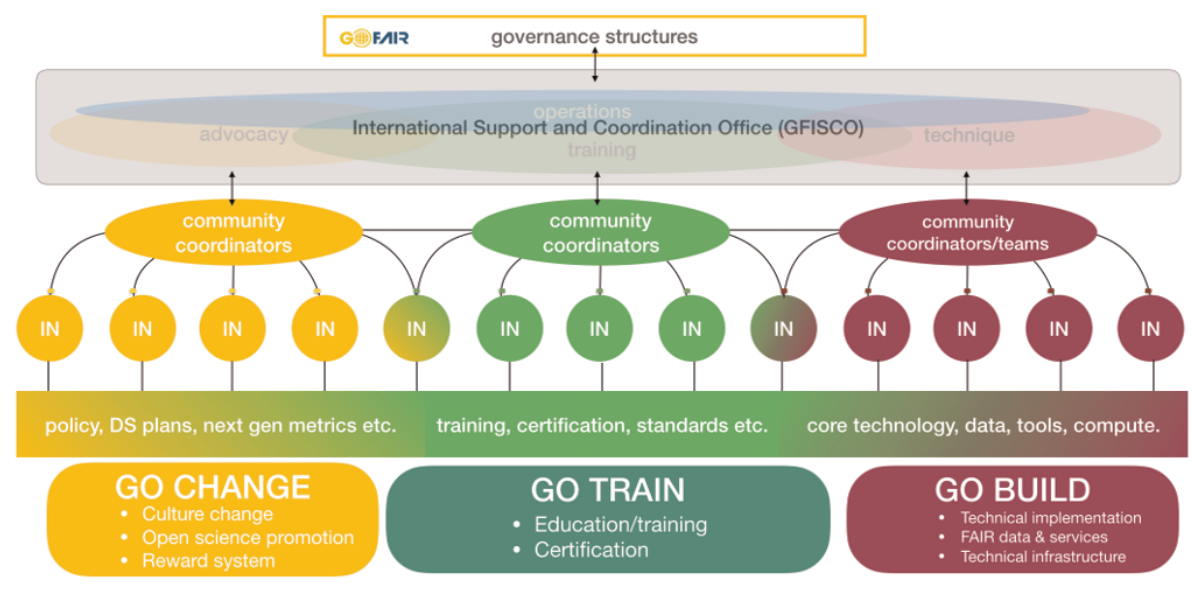

Abb. 1: Die Säulen-Struktur der GO FAIR-Initiative ${ }^{12}$

11 S. https://www.zbw.eu/de.

12 Grafik vom internationalen GO FAIR-Koordinations- und Unterstützungsbüro zur Verfügung gestellt. 


\section{Die GO FAIR-Implementierungsnetzwerke}

Die IN der GO FAIR-Initiative bearbeiten die themen- und disziplinenspezifischen Probleme bei der Umsetzung der FAIR-Prinzipien im Forschungsprozess. Sie sollen Lösungen und Standards erarbeiten, die von den bestehenden Infrastrukturen und Forschungscommunities angeboten und etabliert werden. Damit sind die IN ein Kernelement bei der Verbreitung der FAIR-Prinzipien.

Zur Gründung eines IN gibt es zwei Möglichkeiten: Interessierte treten entweder einem bestehenden Netzwerk bei oder sie gründen ein eigenes. Um einen aktuellen Überblick über bereits bestehende IN zu erhalten, lohnt sich ein Besuch der GO FAIR-Website. ${ }^{13}$ Mit einem Klick auf eines der hier verlinkten IN lassen sich Informationen zu deren Betätigungsfeldern und Zielen abrufen. Derzeit gibt es knapp 20 aktive IN, die zumeist disziplinäre Communities repräsentieren (wie z. B. Chemie, Materialwissenschaft oder Wirtschafts- und Sozialwissenschaften). Über ein OnlineFormular besteht die Möglichkeit, mit den Koordinatorinnen und Koordinatoren des jeweiligen IN in Kontakt zu treten. Stellt sich dabei heraus, dass die Aktivitäten bzw. Ziele der Interessierten sich mit denen des IN überschneiden, können die Koordinatorinnen und Koordinatoren sie ganz formlos in das Netzwerk aufnehmen entweder als individuelle Mitglieder oder als Repräsentantinnen bzw. Repräsentanten ihrer Organisation.

Mit dem Beitritt zu einem GO FAIR-Implementierungsnetzwerk erkennen Neumitglieder die Verhaltensregeln („Rules of Engagement“14) der GO FAIR-Initiative an. Diese besagen beispielsweise, dass es bei der Mitgliederaufnahme keine Diskriminierung geben darf, dass die Initiative von ihren Mitgliedern selbst verwaltet wird und dass eine Mitgliedschaft bei GO FAIR nicht zu Lobbyzwecken missbraucht werden darf. Jedes IN erklärt in einem sogenannten „Manifest“, dass seine Mitglieder diese Verhaltensregeln anerkennen. Das Manifest besteht aus einem zwei- bis dreiseitigen Dokument, welches darüber hinaus Angaben über den Zweck und die Ziele eines IN enthält und in dem auch sämtliche Mitglieder aufgeführt werden.

Möchte eine Gruppe von Interessierten ein neues IN zu einem Themengebiet gründen, das in der GO FAIR-Community bislang noch nicht bearbeitet wird, so muss sie ein solches Manifest erstellen. Dabei liefert das GO FAIR-Büro Unterstützung, zum Beispiel in Form einer Vorlage, die ebenfalls auf der Website zu finden ist. ${ }^{15}$ Außerdem besteht die Möglichkeit, das Manifest im Rahmen einer Schreibwerkstatt („Manifesto Writing Workshop“) zu verfassen, die einmal im Monat am ZBW - Leibniz-Informationszentrum Wirtschaft in Hamburg angeboten wird. Sobald die Interessengruppe sich auf die Inhalte ihres Manifests geeinigt und eine oder meh-

13 S. https://www.go-fair.org/implementation-networks/overview.

14 S. https://www.go-fair.org/resources/rules-of-engagement.

15 S. https://www.go-fair.org/manifesto-template. 
rere Koordinatorinnen bzw. Koordinatoren bestimmt hat, reicht sie das Dokument beim GO FAIR-Büro ein, wo es dann von den Direktorinnen und Direktoren des Büros offiziell bestätigt wird. Von diesem Zeitpunkt an wird das IN auf der GO FAIR-Website als „aktives Implementierungsnetzwerk“ gelistet und seine Mitglieder können an übergreifenden GO FAIR-Veranstaltungen teilnehmen und in Zusammenarbeit mit dem GO FAIR-Büro eigene themenspezifische Veranstaltungen planen.

\section{GO CHANGE - GO TRAIN - GO BUILD}

Zur Umsetzung der FAIR-Prinzipien wird zurzeit ein Netzwerk aus länder- und disziplinübergreifenden Implementierungsprojekten aufgebaut, so dass Synergien geschaffen und folglich redundante Arbeiten innerhalb der drei Handlungsfelder vermieden werden. Sämtliche Aktivitäten der GO FAIR-Initiative unterstützen die Philosophie von Open Science, um u.a. Forschungsdaten oder Softwarecode wann immer möglich - offen zugänglich zu machen und zur Nachnutzung zur Verfügung zu stellen. Als Vorteile werden dadurch Forschungsergebnisse transparent, weitere Forschung wird kostengünstig ermöglicht, Qualitätssicherung kann betrieben und Vertrauen in die Wissenschaft hergestellt bzw. aufrechterhalten werden. Somit ebnet die GO FAIR-Initiative sowohl den Weg zur NFDI als auch zur EOSC, welche den wissenschaftlichen Fortschritt und eine interdisziplinäre Forschung zukünftig in Deutschland und ganz Europa vorantreiben werden.

Um das Wissenschaftssystem auf allen Ebenen zu durchdringen, sind Anstrengungen in verschiedenen Handlungsfeldern notwendig. Innerhalb der GO FAIR-Initiative werden diese als GO CHANGE, GO TRAIN und GO BUILD bezeichnet (s. Abb. 2).

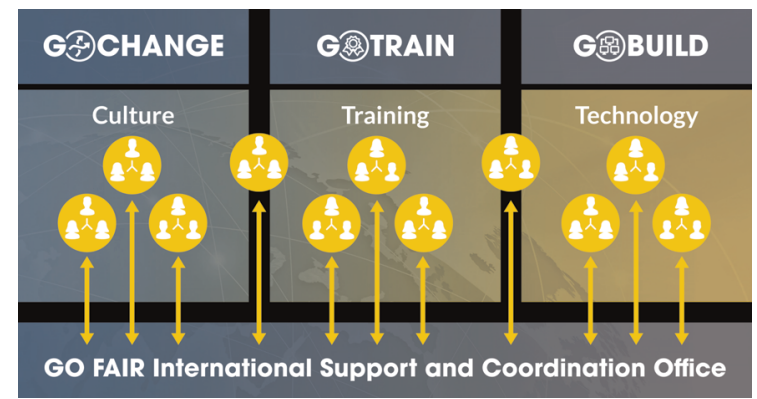

Abb. 2: Die drei Handlungsfelder der GO FAIR-Initiative ${ }^{16}$

16 https://www.go-fair.org/go-fair-initiative/. 
GO CHANGE zielt auf den kulturellen Wandel ab, der die FAIR-Prinzipien zu einem normativen Referenzrahmen für den Umgang mit Forschungsdaten in den Wissenschaften macht. Hierdurch sollen Belohnungssysteme reformiert werden, so dass offene wissenschaftliche Aktivitäten einbezogen und die den Forschungsergebnissen zugrundeliegende Daten zukünftig als ein wichtiges Ergebnis des Forschungsprozesses anerkannt werden.

GO TRAIN beschäftigt sich mit der Aus- und Weiterbildung erforderlicher Datenexpertinnen und -experten. Bereits vor Beginn eines Forschungsprojektes muss mittels eines Datenmanagementplans eine aussagekräftige Beschreibung der Forschungsdaten geplant werden, so dass die Forschungsdaten nach Abschluss des Projektes nachnutzbar sind. Für diesen verantwortungsvollen Umgang ist die Vermittlung von Kernkompetenzen im Bereich Datenadministration (Data Stewardship) elementar. Die Datenadministration nimmt eine Vermittlungsposition zwischen Wissenschaft und Infrastrukturentwicklern ein.

GO BUILD befasst sich mit dem Bedarf an interoperablen und föderierten Forschungsdateninfrastrukturen. Hierdurch wird eine Harmonisierung von Standards, Protokollen und Diensten angestrebt, die es allen Forschenden ermöglicht, wissenschaftliche Daten fachübergreifend zu hinterlegen, abzurufen, zu verknüpfen und $\mathrm{zu}$ analysieren.

Dem Handlungsfeld GO CHANGE kommt bei der praktischen Umsetzung der FAIR-Prinzipien eine besondere Rolle zu. Denn obwohl die Notwendigkeit zum Data Sharing mittlerweile von vielen Forschenden und forschungsfördernden Einrichtungen anerkannt wird, bleibt ein übergreifender Mentalitätswechsel bzw. Kulturwandel diesbezüglich aus. Nach wie vor verhalten sich die meisten Forschenden eher zurückhaltend, wenn es um das Teilen der eigenen Daten geht, ${ }^{17}$ in den Communities gab es - initiiert unter anderem durch die Deutsche Forschungsgemeinschaft (DFG) - eine Auseinandersetzung, die zur Aufnahme eines transparenten FDM in den Kanon guter wissenschaftlicher Arbeit führte. ${ }^{18}$ Diese deutsche Dynamik nimmt GO CHANGE mit einem europäischen Anspruch auf. Im Folgenden sollen die Relevanz von GO CHANGE und die damit verbundenen Bemühungen behandelt werden.

17 Vgl. Linne und Zenk-Möltgen 2017.

18 Vgl. DFG 2019, Leitlinie 13, 17. 


\section{Data Sharing aktuell}

Auch wenn innerhalb der letzten Jahre weltweit eine steigende Bereitschaft seitens der Forschenden zum Data Sharing zu verzeichnen ist, ${ }^{19}$ konnte sich im deutschen Wissenschaftssystem bisher keine flächendeckende Data-Sharing-Kultur und eine daraus resultierende selbstverständliche FDM-Praxis durchsetzen. Dies bestätigt u. a. das vom BMBF geförderte Verbundprojekt UNEKE, ${ }^{20}$ in dessen Rahmen von 2017 bis 2019 eine Umfrage zur Bedarfsanalyse eines zukunftsgerichteten FDM für Institutionen an deutschen Hochschulen durchgeführt wurde. Hieran nahmen insgesamt 1684 Personen von 13 Hochschulen aus allen Forschungsbereichen teil. Über die Hälfte aller Befragten gab an, ihre Forschungsdaten nach Erstverwertung nicht veröffentlichen oder teilen zu wollen, obwohl die Mehrwerte einer offenen Datenkultur bekannt sind. Lediglich vier Prozent der Forschenden nutzten Forschungsdatenrepositorien (FDR) oder Datenzentren zur Archivierung oder Veröffentlichung ihrer Daten und ebenso wenige Personen verwendeten allgemeine oder fachspezifische Metadatenstandards. ${ }^{21}$ Dieser Umstand ist insbesondere vor dem Hintergrund kritisch zu betrachten, als die Verwendung von Repositorien und standardisierter Metadaten unerlässlich ist, um Forschungsdaten im Sinne der FAIRPrinzipien auffindbar, zugänglich, interoperabel und nachnutzbar zu machen.

Dieser Trend deckt sich mit den Informationen des Open Science Monitors (OSM) der Europäischen Kommission, ${ }^{22}$ der die Entwicklungen und Aktivitäten der Open-Science-Bewegung europaweit und disziplinübergreifend beobachtet. In Europa wird das Teilen von Forschungsdaten laut OSM vorrangig zwischen Forschenden praktiziert, die im selben Projekt arbeiten oder sich persönlich kennen. Eine Weitergabe an Forschende, die persönlich nicht bekannt sind, fand im Jahr 2016 nur in 14 Prozent aller erhobenen Fälle statt und zwei Jahre später sogar nur noch in elf Prozent (s. Abb. 3).

19 Vgl. Vocile 2017.

20 Die Abkürzung UNEKE ist das Akronym für „Vom USB-Stick zur NFDI - Entwicklung eines Kriterien geleiteten Entscheidungsmodells für den Aufbāu von Forschüngsdateninfrastrukturen“; s. https://uneke.de/.

21 Vgl. Rehwald und Brenger 2019.

22 S. https://ec.europa.eu/info/research-and-innovation/strategy/goals-research-and-innovationpolicy/open-science/open-science-monitor_en. 


\section{Sharing of research data: \% of researchers that have directly shared research data from their last project, by recipient Reference dates: 2016 and 2018}

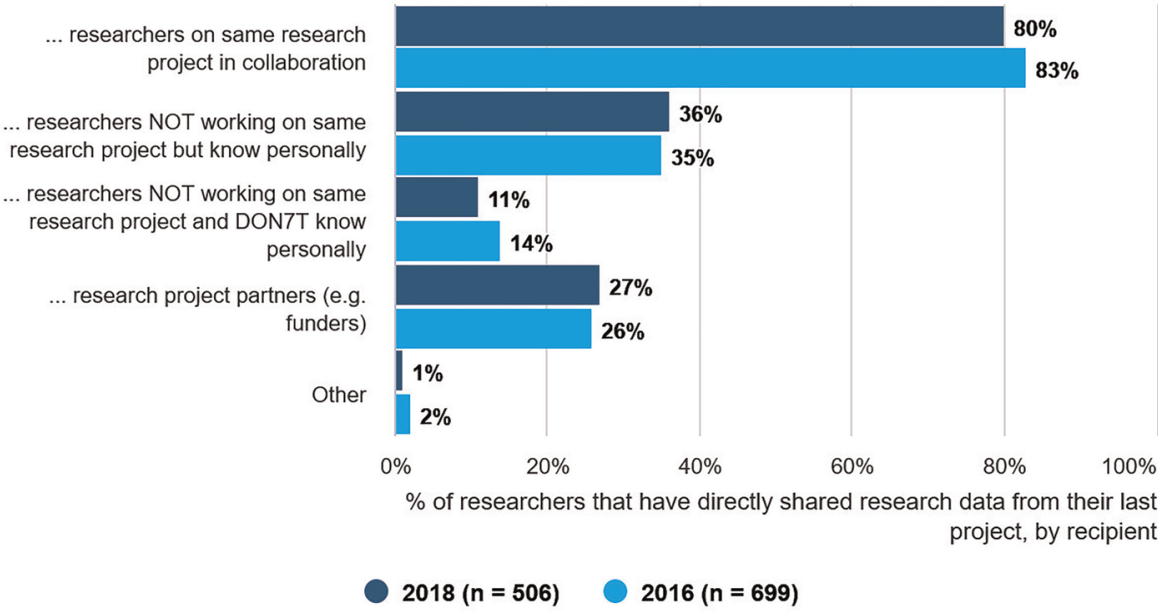

Abb. 3: Data Sharing nach Empfänger 2016 und 2018 (Open Science Monitor) ${ }^{23}$

Im OSM werden keine Gründe für diese Zurückhaltung angegeben. Solche sind allerdings im Wiley Open Science Researcher Survey $2016^{24}$ aufgeführt, in dem weltweit 4600 Autorinnen und Autoren in 112 Ländern zu ihrem Data-Sharing-Verhalten befragt wurden. Der Studie gemäß sind die vier meistgenannten Gründe, warum Forschende zögern, ihre Forschungsdaten zu teilen, die folgenden:

- $50 \%$ - "Intellectual property or confidentiality issues" 25

- $31 \%$ - "Ethical concerns" 26

- $\quad 23 \%$ - "I am concerned about misinterpretation or misuse of my research"27

- $22 \%$ - "I am concerned that my research will be scooped"28

Die Hälfte aller Befragten geht davon aus, dass ihre Erhebungsdaten aus datenschutz- und urheberrechtlichen Gründen nicht veröffentlicht werden dürfen und beinahe jede/jeder Dritte gibt ethische Bedenken als Grund an. In den meisten Fäl-

23 https://ec.europa.eu/info/research-and-innovation/strategy/goals-research-and-innovation-policy/open-science/open-science-monitor/facts-and-figures-open-research-data_en.

24 S. https://doi.org/10.6084/m9.figshare.4748332.

25 Vocile 2017.

26 Vocile 2017.

27 Vocile 2017.

28 Vocile 2017. 
len ist hierfür eine Unkenntnis bezüglich datenschutz- und urheberrechtlicher Anforderungen verantwortlich zu machen, was nicht zuletzt daran liegt, dass die diesbezügliche Rechtslage einerseits sehr komplex und andererseits in Teilen ungeklärt ist. Dies führt sowohl zu Unsicherheiten als auch zu Fehlverhalten seitens der Forschenden. Beispielsweise sind Datenschutzformulierungen in informierten Einwilligungen oftmals aus Unwissenheit zu restriktiv, wodurch Nachnutzungsmöglichkeiten unnötigerweise eingeschränkt oder gar komplett ausgeschlossen werden. ${ }^{29}$

Neben der rechtlichen Unsicherheit spielen mangelndes Vertrauen und eine von Konkurrenz geprägte Forschungsmentalität eine relevante Rolle. Die Forschenden haben Sorge vor fehlerhaft durchgeführten Replikationsstudien und daraus folgenden Reputationsverlusten oder aber auch Missbrauch ihrer Forschung. Darüber hinaus befürchten sie, dass andere ihre Forschungsdaten zum eigenen Reputationsgewinn verwenden könnten, ohne dass ein Reputationstransfer auf die Primärforschenden erfolgt. ${ }^{30}$

Auf die bekannten Vorteile des Data Sharings soll an dieser Stelle nicht mehr eingegangen werden. Vielmehr sollen die Chancen hervorgehoben werden, welche die GO FAIR-Initiative insbesondere mit ihrem Handlungsfeld GO CHANGE hinsichtlich eines Kulturwandels zum Data Sharing im Wissenschaftssystem mit sich bringen.

\section{Die besondere Rolle von GO CHANGE}

Dem Handlungsfeld GO CHANGE kommt innerhalb der GO FAIR-Initiative, aber insbesondere über die Initiative hinaus, eine wesentliche Rolle zu. Aktuell fehlt ein funktionierendes Belohnungs- und Anreizsystem für ein effizientes Data Sharing, welches den notwendigen Kulturwandel unterstützen und beschleunigen könnte. An dieser Stelle setzt GO CHANGE an:

GO CHANGE zielt auf den kulturellen Wandel, der die FAIR-Prinzipien zu einem funktionierenden Standard in der Wissenschaft macht und die Belohnungssysteme so reformiert, dass offene wissenschaftliche Aktivitäten einbezogen werden und Forschungsdaten zukünftig als ein wichtiges Ergebnis des Forschungsprozesses anerkannt werden. ${ }^{31}$

Aus diesem Grunde wurde die Säule GO CHANGE innerhalb der GO FAIR-Initiative eingerichtet. Die Bemühungen in GO CHANGE sollen die Koordination zwischen vorhandenen FDM-Projekten optimieren und weitere Aktivitäten einleiten, welche langfristig zum kulturellen Wandel und einem daraus resultierenden Mentalitäts-

29 Vgl. Droß und Naujoks 2019, 28-30.

30 S. a. Beitrag von Oßwald, Kap. 3.5 in diesem Praxishandbuch.

31 ZBW 2019. 
wandel der Forschenden und ihrer Communities führen sollen. Innerhalb der Säule wurden u. a. folgende Ziele, Werte und Aktivitäten zur Einleitung eines Paradigmenwechsels in Bezug auf FDM innerhalb des Wissenschaftssystems formuliert:

- Vermittlung und Verankerung der Vorteile des FDM und Data Sharings in den wissenschaftlichen Communities.

- Entwicklung eines funktionierenden Belohnungs- und Anreizsystems für ein besseres FAIR-Datenmanagement.

- Gemeinsame Unterstützung und Umsetzung der FAIR Prinzipien für Forschungsdaten, Algorithmen, Prozesse, Software, etc.

- Verhinderung weiterer Fragmentierung der Forschungsdatenlandschaft und Förderung von Aktivitäten zur aktiven Föderierung.

- Zusammenarbeit zur Sicherung der technischen Kompatibilität von FDM-Tools.

- Vernetzung und Sichtbarkeit durch das GO FAIR-Büro: Arbeitsgruppentreffen, regelmäßige Workshops, Unterstützung bei öffentlichkeitswirksamen Dokumenten (Best-Practices, Ergebnisberichte).

- Förderung von Open Science.

- Förderung von Data Stewardship und der damit einhergehenden Interessenvertretung.

- Vermittlung von Best Practices zur Erstellung von Datenmanagementplänen und deren Umsetzung im Forschungsprojekt.

Das übergeordnete Ziel der GO CHANGE-Säule besteht darin, Unterstützung und Anleitung zur Erreichung dieser Ziele bereitzustellen. Hierbei hat der Kulturwandel Vorrang, in dem Daten als eigenständige Forschungsleistung statt lediglich als Nebenprodukt von Forschungsprojekten betrachtet werden. Um dies $\mathrm{zu}$ erreichen, muss die zumeist nicht standardisierte Datenverwaltung innerhalb von Forschungsprozessen in ein professionelles und nachhaltiges FDM transformiert werden, welches den Datenzugang zur Sekundärverwertung im Sinne der FAIR-Prinzipien erst ermöglicht.

Im Prozess des Paradigmenwechsels spielen nicht nur Forschende und wissenschaftliche Einrichtungen, sondern auch Förderorganisationen eine zentrale strategische Rolle. Da bei den Forschenden bisher keine intrinsische Motivation, weder zum FDM noch zum Data Sharing übergreifend zu erkennen ist, muss zunächst eine extrinsische Motivationsquelle geschaffen werden. Diese kann beispielsweise in Form finanzieller Unterstützung des FDM oder restriktiveren FDM-Anforderungen seitens der Forschungsförderer realisiert werden. Sobald sich ein Reputationsgewinn durch das Publizieren von Forschungsdaten durchgesetzt hat (bzw. das Zurückhalten von Daten zu einem Reputationsverlust führt), ist zu erwarten, dass sich die Motivation zum FDM und Data Sharing ausweiten und sich ein diesbezüglicher Kulturwandel dynamisiert. 


\section{Implementierungsnetzwerke in GO CHANGE}

Was sind die konkreten Themen, welche die IN innerhalb der Säule GO CHANGE bearbeiten? Um den kulturellen Wandel in Richtung FAIRes FDM voranzutreiben, setzen die IN meist an mehreren Hebeln an. Dabei überschneiden sie sich durchaus in den von ihnen geplanten Aktivitäten, was wiederum Synergiepotenzial birgt. Einen Überblick über die verschiedenen Tätigkeitsbereiche und darin involvierten IN gibt Abb. 4 .

Als graue Kreise abgebildet sind in Abb. 4 die 15 aktiven IN, die sich zum Zeitpunkt des Verfassens dieses Artikels mit Herausforderungen des kulturellen Wandels beschäftigen. Von den grauen Kreisen gehen Pfeile ab, die jeweils eine Tätigkeit des IN beschreiben. Sie münden entweder in einen hellgrünen Kreis oder einen orangen Kreis. Hellgrüne Kreise kennzeichnen Themen, die von mehreren IN behandelt werden. Bei den orangen Kreisen handelt es sich um Themen, die (bisher) nur von einem einzelnen IN bearbeitet werden. Im weiteren Verlauf dieses Unterkapitels wird auf die Schnittmengen nacheinander eingegangen und erläutert, wie diese von den IN bearbeitet werden.

\section{Bewusstsein für FAIR-Prinzipien}

Zehn IN haben es sich zur Aufgabe gemacht, ihre Communities für die FAIR-Prinzipien zu sensibilisieren. Das IN „EcoSoc“32 beispielsweise, welches als Praxisbeispiel in Abschnitt 7 dieses Beitrags ausführlich vorgestellt wird, möchte besonders in den Wirtschafts- und Sozialwissenschaften ein Bewusstsein für das Teilen von Forschungsdaten schaffen, z. B. durch die Stärkung des Modells der Forschungsdatenzentren. Ein ähnliches Ziel verfolgt NOMAD innerhalb der Materialwissenschaften. Dabei stellt sich die Frage, wie genau ein solches Bewusstsein unter Forschenden befördert werden kann. Welche Formate, Materialien und Angebote funktionieren in einer bestimmten Community oder auch disziplinübergreifend? $\mathrm{Zu}$ genau diesen Fragen veranstaltete das GO FAIR-Büro im Juni 2019 einen GO CHANGE Workshop, bei dem sich Mitglieder von bestehenden und interessierten IN über ihre Ansätze, Erfahrungen und Best Practices austauschen konnten. Ein Ergebnis des Workshops ist $\mathrm{u}$. a. eine Sammlung von Awareness-Materialien, die nun in einer Datenbank aufbereitet zur Nachnutzung zur Verfügung stehen. ${ }^{33}$ Darüber hinaus wurde im Rahmen des Workshops das IN „GO UNI“ initiiert. GO UNI soll universitäre FDM-Kompetenzzentren und im FDM-Kontext aktive Einrichtungen miteinander vernetzen, um Synergien bei der Umsetzung und Etablierung der FAIR-Prinzipien nutzen zu können. Vorrangiges Ziel ist eine gegenseitige Unterstützung zum Aufbau notwendiger institutioneller Strukturen für ein professionelles FDM.

32 S. https://www.go-fair.org/implementation-networks/overview/ecosoc-in/.

33 S. https://www.go-fair.org/resources/more-on-fair. 


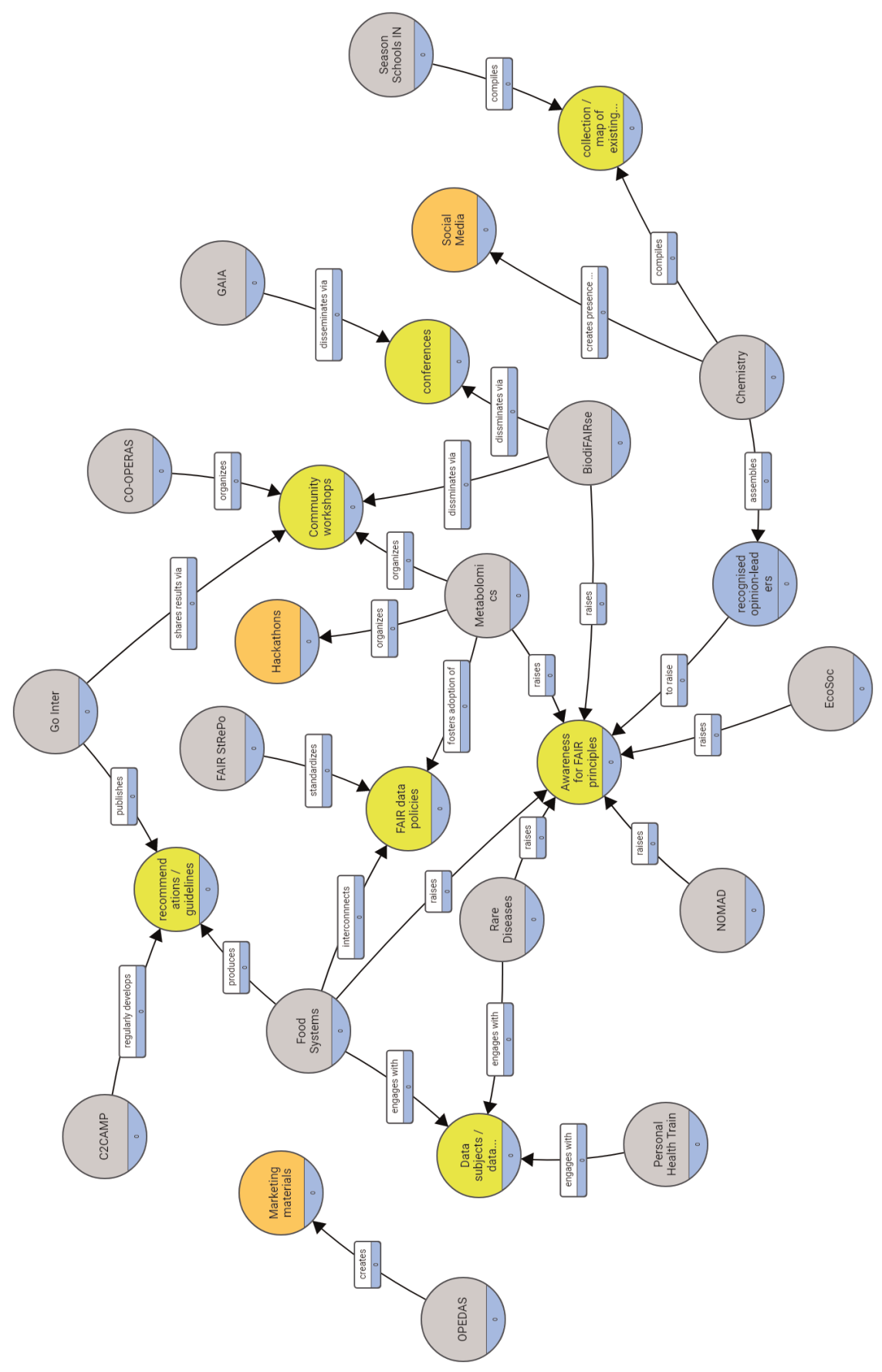

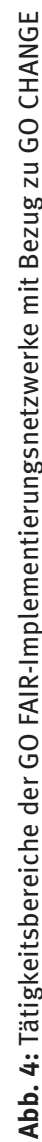




\section{Community Workshops}

Vier der IN stellen in ihren Manifesten heraus, dass sie spezielle Workshops durchführen möchten, um die breitere Forschungsgemeinschaft rund um ihren Tätigkeitsbereich einzubeziehen. Das IN „CO-OPERAS“34 nutzte hierfür die Open Science Fair 2019 in Porto als Plattform, um einen Workshop für die Forschungscommunity der Sozial- und Geisteswissenschaften anzubieten. Dabei befassten sie sich insbesondere mit der disziplinspezifischen Herausforderung der Mehrsprachigkeit bei der Umsetzung der FAIR-Prinzipen.

Das IN „Go Inter“35 wiederum arbeitet schwerpunktmäßig an der Realisierung semantischer Interoperabilität über Disziplingrenzen hinweg. Es plant Workshops mit Vertretenden verschiedener Disziplinen, aus denen sich seine Anwendungsfälle speisen. Bei der Organisation solcher Workshops können die IN auf die Unterstützung des GO FAIR-Büros zurückgreifen, zum Beispiel in Form von Reisemittelzuschüssen für die Teilnehmenden.

\section{Datensubjekte/Datenlieferanten}

In den Wandel hin zu einer FAIRen Datenkultur möchten drei IN auch Datensubjekte miteinbeziehen, also diejenigen, von denen personenbezogene Daten erhoben werden. Sowohl im IN „Personal Health Train“36, als auch bei „Rare Diseases“37 geht es dabei um persönliche Gesundheitsdaten. „Personal Health Train“ möchte solche sensiblen Gesundheitsdaten mithilfe einer föderierten Infrastruktur sicher nachnutzbar machen. Dabei sollen Bürgerinnen und Bürger genau kontrollieren können, welche Daten sie für wissenschaftliche Analysen zur Verfügung stellen und welche nicht.

Das IN „Rare Diseases“ möchte auf Grundlage FAIRer Daten schnellere Diagnosen und bessere Behandlungsmöglichkeiten von seltenen Krankheiten erwirken. Wie genau die FAIR-Prinzipien in diesem Fall umgesetzt werden, sollen auch Patientenorganisationen mitbestimmen. Das GO FAIR-Büro unterstützt die IN bei der Einbindung solcher Interessensgruppen und hilft z. B. bei der zielgruppengerechten Formulierung von Informationsmaterialien.

34 S. https://www.go-fair.org/implementation-networks/overview/co-operas/.

35 S. https://www.go-fair.org/implementation-networks/overview/go-inter/.

36 S. https://www.go-fair.org/implementation-networks/overview/personal-health-train/.

37 S. https://www.go-fair.org/implementation-networks/overview/rare-diseases/. 


\section{Empfehlungen/Guidelines}

Praktische Empfehlungen und Guidelines zum FDM werden von drei IN erstellt und veröffentlicht. Das IN „Food Systems“38 etwa möchte auf Empfehlungen von Arbeitsgruppen der Research Data Alliance (RDA) ${ }^{39}$, der Initiative Global Open Data for Agriculture and Nutrition ${ }^{40}$ und dem World Wide Web Consortium ${ }^{41}$ aufbauen und diese, wenn nötig, um spezifische Aspekte des FAIRen FDM in der Lebensmittelsystemforschung erweitern. Bei der Produktion und Verbreitung entsprechender Dokumente ist das GO FAIR-Büro den IN behilflich.

\section{Daten-Policies}

Drei IN nehmen in ihrem Manifest Bezug auf Daten-Policies, also institutionelle Verhaltensregeln für den Umgang mit Forschungsdaten. Das IN „FAIR StRePo“42 (kurz für „FAIR Standards, Repositories, and Policies“) steht in engem Zusammenhang mit der Plattform fairsharing.org, ${ }^{43}$ welche Daten-Policies unterschiedlichen Ursprungs (Forschungsförderer, Fachgesellschaften, Fachzeitschriften etc.) sammelt, systematisiert und zugänglich macht. Innerhalb der GO FAIR-Initiative möchte das IN „FAIR StRePo“ diejenigen Daten-Policies, welche durch andere IN erstellt werden oder auf deren Grundlage sie selber handeln, bekannter machen, indem sie diese auf fairsharing.org veröffentlicht. Formulierungshilfen zur Erstellung institutioneller Daten-Policies hat das GO FAIR-Büro in seiner Datenbank ${ }^{44}$ gesammelt.

\section{Konferenzen}

Ein Bewusstsein für FAIRes Datenmanagement möchten zwei IN ausdrücklich auf Konferenzen schaffen. Das IN „GAIA“45, das in der Erdsystemforschung verankert ist, nimmt hierfür insbesondere die großen internationalen Konferenzen ihrer Forschungscommunity ins Visier. Hier soll es in Bezug auf FAIR Data vor allem darum gehen, dass sich die internationale Forschungscommunity auf bestimmte Metadatenstandards verständigt und diese Einigungen gesteigerte Aufmerksamkeit und

38 S. https://www.go-fair.org/implementation-networks/overview/food-systems/.

39 S. https://www.rd-alliance.org/.

40 S. https://www.godan.info/.

41 S. https://www.w3.org/.

42 S. https://www.go-fair.org/implementation-networks/overview/fair-strepo/.

43 S. https://fairsharing.org/.

44 S. https://www.go-fair.org/resources/more-on-fair.

45 S. https://www.go-fair.org/implementation-networks/overview/gaia-data/. 
weitere Akzeptanz erhalten. Auf anstehende Konferenzbeiträge von IN weist das GO FAIR-Büro in einem alle zwei Monate erscheinenden Newsletter hin. ${ }^{46}$

\section{Ressourcenübersicht}

Zwei IN planen, eine Übersicht über schon existierende Ressourcen im Zusammenhang mit FAIRem FDM zu erstellen. Beim IN „Chemistry“47 handelt es sich dabei z. B. um in der Chemie und ihren Teildisziplinen aufgesetzte Standards zur Benennung chemischer Stoffe. Diese möchte das IN sammeln und von „FAIR StRePo“ kuratieren lassen. Dem im Aufbau befindlichen IN „Season Schools“ wiederum geht es darum, ein Verzeichnis bestehender Schulungsprogramme zu FAIRem FDM inklusive der dazugehörigen Trainerinnen bzw. Trainer und Organisationen zu erstellen. Die von den IN erstellten Übersichten lassen sich einfach in der vom GO FAIR-Büro aufgesetzten Datenbank ${ }^{48}$ integrieren, so dass sie eine größere Reichweite erzielen.

\section{EcoSoc - FAIR-Prinzipien in den Wirtschafts-, Verhaltens,- und Sozialwissenschaften etablieren}

Das Economic and Social Sciences goINg FAIR Implementation Network (EcoSocIN $)^{49}$ ist das erste GO FAIR IN für die Sozial-, Verhaltens- und Wirtschaftswissenschaften. EcoSoc-IN wurde im November 2018 auf Initiative des Rates für Sozialund Wirtschaftsdaten (RatSWD) ${ }^{50}$ gegründet und basiert auf der langjährigen Expertise des RatSWD und der durch ihn akkreditierten Forschungsdatenzentren (FDZ). ${ }^{51}$ Der RatSWD berät seit 2004 die Bundes- und Landesregierungen bei der Verbesserung der Forschungsdateninfrastruktur für die Sozial-, Verhaltens- und Wirtschaftswissenschaften. Nachweislich verbessert haben sich durch seine Bemühungen bereits der Zugang zu sensitiven Forschungsdaten der amtlichen Statistik und amtli-

46 Abonnement unter https://www.go-fair.org/newsletter-subscription.

47 S. https://www.go-fair.org/implementation-networks/overview/chemistryin/.

48 S. https://www.go-fair.org/resources/more-on-fair.

49 S. https://www.go-fair.org/implementation-networks/overview/ecosoc-in/.

50 S. https://www.ratswd.de/.

51 S. https://www.ratswd.de/forschungsdaten/fdz. 
chen Registern, ${ }^{52}$ der Sozialversicherungsträger, ${ }^{53}$ Ressortforschungseinrichtungen ${ }^{54}$ und wissenschaftlicher Forschungsinstitute die insbesondere die Daten der großen Umfragestudien ${ }^{55}$ vorhalten. ${ }^{56}$ Insgesamt haben sich mittlerweile (Stand Juni 2020) 38 FDZ durch den RatSWD akkreditieren lassen (siehe Abb. 5) und garantieren damit mindestens einen Zugangsweg für die Nachnutzung der von ihnen kuratierten Forschungsdaten. Darüber hinaus entstehen in diesem Vernetzungskontext auch Repositoriumservices insbesondere für sensible Daten, die den angestrebten Datenkulturwandel mit einer dafür notwendigen Infrastruktur unterlegen.

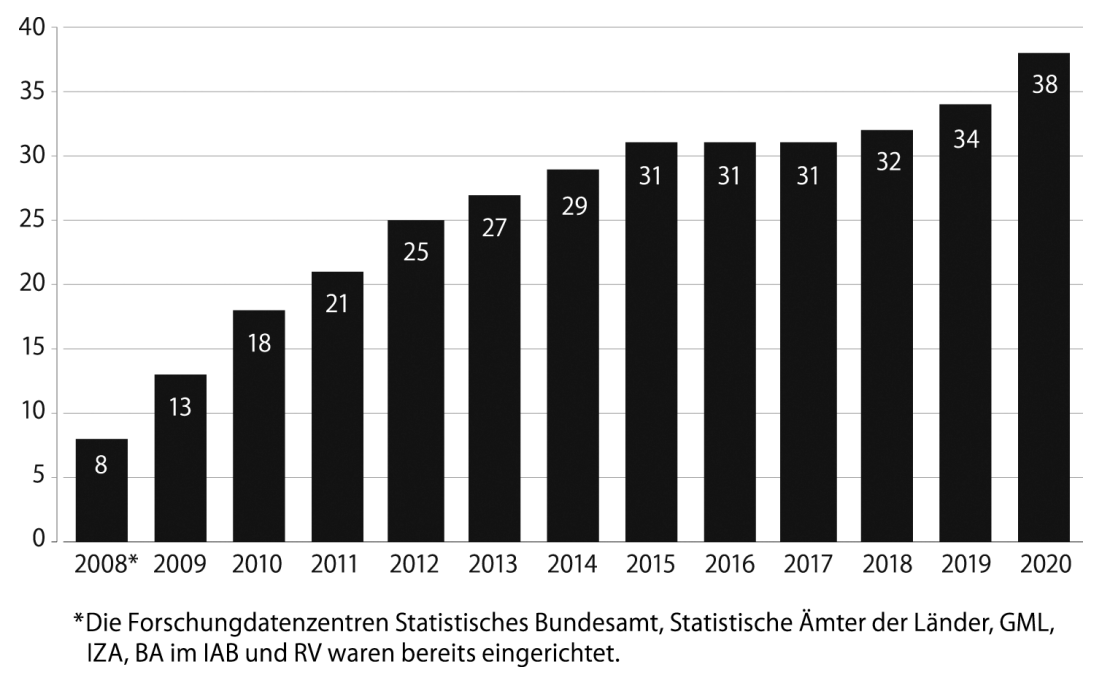

Abb. 5: Die Entwicklung der vom RatSWD akkreditierten Forschungsdatenzentren

Da Forschungsdaten in den Wirtschafts-, Verhaltens-, und Sozialwissenschaften ähnlich wie in der klinischen oder epidemiologischen Forschung - in der Regel schützenswerte Informationen zu konkreten Personen, Unternehmen oder Institutionen enthalten, müssen die rechtlichen Bestimmungen zum Datenschutz und die Zusicherung der Vertraulichkeit eingehalten werden. Solche sensitiven Daten wer-

52 insbesondere den statistischen Ämtern des Bundes und der Länder, Bundesbank, und Kraftfahrtbundesamt.

53 Deutsche Rentenversicherung Bund, Bundesagentur für Arbeit.

54 z. B. Robert-Koch-Institut, Bundeszentrale für gesundheitliche Aufklärung, Bundesinstitut für Berufsbildung, Deutsches Zentrum für Altersfragen, Deutsches Jugendinstitut.

55 z. B. Survey of Health, Ageing and Retirement, Sozioökonomisches Panel, Nationales Bildungspanel, Internationale Umfrageprogramme wie European Social Survey.

56 Vgl. Bug et al. 2018, 574-579; RatSWD 2019b, die URLs der FDZ sind abrufbar über: https:// www.ratswd.de/forschungsdaten/fdz. 
den deshalb für das Teilen von Daten anonymisiert, indem Attribute vergröbert oder aus den Daten entfernt werden. Je stärker die Anonymisierung ist, desto besser sind die Teilnehmenden der Studien geschützt. Gleichzeitig wird das analytische Potenzial der Daten verringert. Werden zum Beispiel Informationen zum Wohnort der Teilnehmenden in einer Befragung entfernt, ist es, bei Fehlen weiterer personenbezogener Informationen, praktisch nicht mehr möglich, die Person zu re-identifizieren. Allerdings können auch keine Einflüsse des Wohnorts auf Einstellungen und Verhalten der Individuen untersucht werden. Im Fall von personenbezogenen und anderen vertraulichen Daten muss bei der Anonymisierung deshalb zwischen Schutzinteressen der Studienteilnehmenden und Interessen der Forschenden abgewogen werden. Nicht alle Daten können als Open Data frei zur Verfügung gestellt werden. Vielmehr müssen die Zugangswege für Forschende dem Grad der Anonymisierung entsprechend ausgestaltet werden. Die FDZ leisten diesen Abwägungsprozess und bemühen sich im Sinne des Konzepts der „intelligent openness“, ${ }^{77}$ den Zugang so offen wie möglich, aber so sicher wie nötig zu gestalten. Der Zugang zu Forschungsdaten - im Sinne der accessibility - ist folglich das Kernthema des RatSWD und die Kernkompetenz der FDZ. Mit der flächendeckenden Einführung von Digital Object Identifiers (DOI) ${ }^{58}$ und einem umfassenden Nachweissystem für Forschungsdaten wurde auch die übergreifende Suche nach Forschungsdaten innerhalb der FDZ in den vergangenen Jahren vereinfacht und die Auffindbarkeit der Daten gestärkt.
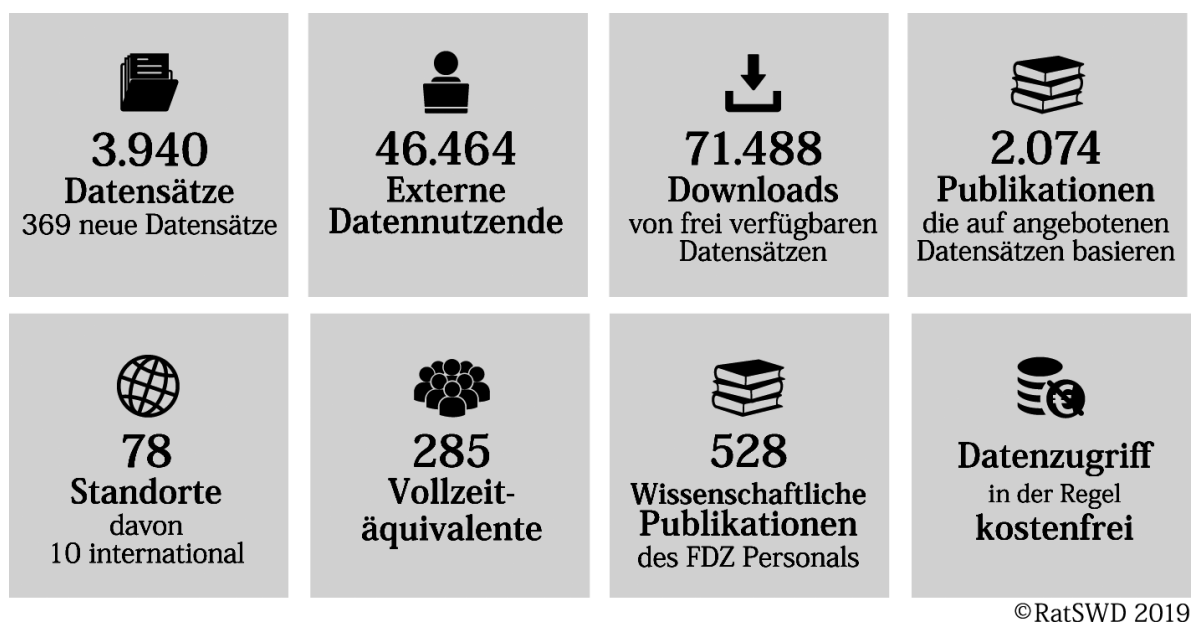

Abb. 6: Kennzahlen des vom RatSWD akkreditierten Netzwerks aus damals 34 Forschungsdatenzentren im Jahr 2018

57 Vgl. Royal Society 2012.

58 S. https://www.doi.org/. 
Von diesem Status quo aus entstand das Manifest des EcoSoc-IN im engen Austausch mit den FDZ, da sie über die Expertise im Datenmanagement verfügen und bereits eine große Nutzendenbasis haben (siehe Abb. 6).

2017 wurde zunächst ein Vergleich der FAIR-Prinzipien mit dem bereits etablierten FDM der FDZ durchgeführt. Diese Evaluation hat gezeigt, dass viele Aspekte in der täglichen Praxis von einem Großteil der FDZ bereits umgesetzt werden, speziell bezüglich der Auffindbarkeit, Zugänglichkeit und Nachnutzbarkeit von Forschungsdaten. Hinsichtlich der Vereinheitlichung von Metadatenstandards, der Verbesserung der Interoperabilität von Daten und bei der Maschinenlesbarkeit besteht jedoch noch Handlungsbedarf, der gleichzeitig den unterschiedlichen disziplinären Anforderungen gerecht werden muss - eine große Herausforderung.

Der konzeptionelle Rahmen der FAIR-Prinzipien bietet eine besonders geeignete Herangehensweise, um einerseits interdisziplinär gültige Prinzipien und andererseits disziplinspezifische Eigenheiten im FDM zu verbinden. Primäres Ziel von EcoSoc-IN ist daher die weitere Verbreitung und spezifische Weiterentwicklung der FAIR-Prinzipien, so dass sie innerhalb der FDZ und darüber hinaus Anwendung finden. Dazu müssen die abstrakten Prinzipien für die Sozial-, Verhaltens- und Wirtschaftswissenschaften ausformuliert werden und dabei die Eigenheiten der verwendeten Datenarten und Objekttypen aufgegriffen werden Dies gilt insbesondere für die FAIR-Prinzipien, die nicht generisch technisch (d.h. Nutzung persistenter Identifier, Schnittstellen für Metadaten, usw.) umgesetzt, sondern inhaltlich ausgefüllt werden müssen. Das betrifft z. B. Konventionen über angemessene Zugangsregeln (Access Policies) im Falle von nicht vollständig anonymisierten/anonymisierbaren Daten, aber auch Fragen der Metadatenqualität zur Erleichterung der Nachnutzung und Verbesserung der Interoperabilität. Dabei stehen vier Problemfelder im Vordergrund:

- FAIRer Zugang zu datenschutzrechtlich oder forschungsethisch sensiblen Daten: Da sensitive Daten nicht frei vertrieben werden können, wurden verschiedene Lösungswege entwickelt. Erstens werden faktisch anonymisierte Scientific Use Files auf Antrag und häufig nach Abschluss eines Nutzungsvertrages vertrieben. Zweitens wurden Gastwissenschaftsarbeitplätze in den FDZ für einen überwachten Datenzugang eingerichtet. Drittens schließlich wurden Verfahren für eine kontrollierte Datenfernverarbeitung (ein sogenanntes Remote Access) eingeführt (RatSWD 2019a). Es fehlt aber eine einheitliche Terminologie im Sinne eines formalen Protokolls, das Rechte und Pflichten von Datengebenden und Datennutzenden definiert (data access protocols).

- Angleichung verwendeter Metadatenstandards: Dies ist ein wichtiger Schritt zur Datendokumentation und Definition verschiedener digitaler Objekttypen und erhöht sowohl die Nachnutzbarkeit als auch die Interoperabilität der Daten. Zwar existiert ein umfassender sozialwissenschaftlicher Metadatenstandard der 
Data Documentation Initiative, nämlich DDI Lifecycle, ${ }^{59}$ dieser wird in der Praxis jedoch noch nicht flächendeckend angewendet und es gibt sehr unterschiedliche Dokumentationstiefen. Da die Dokumentation aufwendig ist, werden nicht für alle Studien alle Attribute (d.h. Variablen in den Datensätzen) nach DDI Standard dokumentiert, sondern z. B. lediglich die Fragebögen für die Nachnutzung zur Verfügung gestellt.

- Interoperabilität von Merkmalen: Vor allem in der Umfrageforschung werden bestimmte Attribute (z.B. sozio-demographische Merkmale der Probanden) oft sehr unterschiedlich erhoben. Um Daten aus unterschiedlichen Quellen gewinnbringend verknüpfen zu können, müssen sie interoperabel sein, indem Ontologien zur Harmonisierung (ex-post oder ex-ante) verwendet werden. Solche Ontologien wurden vor allem für die international vergleichende Forschung entwickelt (z.B. die International Standard Classification of Education ${ }^{60}$ ). Ziel ist die systematische Aufbereitung von Daten und die kontinuierliche Weiterentwicklung solcher Klassifikationen für die Datenerhebung und -dokumentation.

- Maschinenlesbare (Meta-)Daten: Die Metadaten und der Datenzugang sind bisher in der Regel für die Verwendung durch Menschen ausgelegt. Ein Kernelement der FAIR-Prinzipien liegt jedoch gerade in der Bereitstellung von Schnittstellen für die Verarbeitung von (Meta-)Daten durch Maschinen. Zwar können Ausschnitte der Metadaten auf Studienebene über standardisierte Schnittstellen (OAI-PMH) abgerufen werden. Metadaten zu den Attributen - also den Inhalten - der Studien liegen jedoch in der Regel nicht maschinenlesbar vor (z. B. liegen Fragebögen nur in PDF-Dateien vor). Auch der automatisierte Zugriff auf Daten stellt die absolute Ausnahme dar.

Praktisch wird das EcoSoc-IN Minimalstandards für die Erfüllung der FAIR-Kriterien in den Wirtschafts-, Verhaltens-, und Sozialwissenschaften definieren und Vorstellungen dazu entwickeln, wie eine vollständig FAIR-konforme Forschungsdateninfrastruktur aussehen kann. Um Insellösungen zu vermeiden, sollte diese Diskussion nicht auf den Kreis der FDZ, die vom RatSWD akkreditiert sind, begrenzt bleiben. Das Format der IN ermöglicht es, den Kreis über die FDZ hinaus national und international zu erweitern. Deshalb ist eine Beteiligung am EcoSoc-IN für alle Organisationen und Personen offen, die das Manifest mitzeichnen. Derzeit hat EcoSoc-IN 23 Mitglieder, darunter 17 FDZ. Mit dem Deutschen Institut für Erwachsenenbildung $(D I E)^{61}$ hat sich ein erstes institutionelles Mitglied ohne direkte Anbindung zum RatSWD angeschlossen.

59 S. https://ddialliance.org/Specification/.

$60 \mathrm{~S}$. http://uis.unesco.org/en/topic/international-standard-classification-education-isced.

61 S. https://www.die-bonn.de/default.aspx. 
Darüber hinaus bietet die GO FAIR-Initiative die Möglichkeit über disziplinäre Grenzen hinweg vergleichbare Probleme zu diskutieren. Eine inhaltliche Nähe zwischen EcoSoc-In und einschlägigen Initiativen aus der Medizin ist dabei besonders relevant. Das IN „Personal Health Train“ beschäftigt sich beispielsweise mit sensitiven Patientendaten (s. Abschnitt 6). Zudem planen die beiden in der ersten Runde der NFDI Ausschreibung (s. Abschnitt 1) eingereichten Konsortien NFDI4Health und NFDI4Medicine ${ }^{62}$ dem EcoSoc-In beizutreten, um gemeinsam Lösungen für Zugang und Interoperabilität von sensitiven Daten aus Kohorten- und Panelstudien zu entwickeln. Fragen der technischen Weiterentwicklung der Infrastruktur könnten mit den IN aus der Go BUILD Säule diskutiert werden (z. B. dem Go INTER IN).

Angesichts der dynamischen Entwicklung der Forschungsdateninfrastrukturen bieten die FAIR-Prinzipien den Datenzentren folglich eine inhaltliche Orientierung bei der konzeptionellen und technischen Weiterentwicklung der eigenen Angebote. FAIR-konforme Infrastrukturen werden zur Bedingung für die Beteiligung an der EOSC und der NFDI und deshalb setzt die GO FAIR-Initiative mit GO CHANGE und der Umsetzung der FAIR-Prinzipien wichtige Impulse für die Zukunft. Dies erfordert ein offenes Forum für die Diskussion, wie die FAIR-Prinzipien in den Sozial-, Verhaltens- und Wirtschaftswissenschaften umgesetzt werden sollen. Dazu stellt das EcoSoc-IN, aber auch alle anderen IN der GO FAIR Initiative aufgrund ihrer strukturellen Offenheit das geeignete Format dar.

\section{Ausblick: Angestrebte Auswirkungen von GO FAIR und GO CHANGE auf das deutsche Wissenschafts- system}

Science is moving towards a greater openness, in terms of not just data but also publications, computer code and workflows. Yet researchers who are learning to navigate the open-science arena face a thicket of thorny issues. ${ }^{63}$

Die GO FAIR-Initiative möchte alle Open Science-Stakeholder dabei unterstützen, das Dickicht der „Open-Science-Arena“ leichter zu durchdringen und langfristig wirkende FDM-Netzwerke aufzubauen. Die zahlreichen IN, die sich sehr zügig auf Initiative von GO FAIR hin gegründet haben, weisen auf einen solchen Unterstützungsbedarf hin. Gleichzeitig zeigt die Gründung der IN, dass ein Paradigmenwechsel bereits stattfindet, der FDM und Data Sharing langfristig zu einem Standard in

62 S. https://www.dfg.de/download/pdf/foerderung/programme/nfdi/191212_nfdi_statistik_antragseingang.pdf, Anhang Tabelle 4, $17 \mathrm{ff}$.

63 Popkin 2019, 446. 
der Wissenschaft etablieren wird. Innerhalb der wissenschaftlichen Communities sind disziplinübergreifend ein hoher Vernetzungsbedarf und eine große Bereitschaft zur Zusammenarbeit zu verzeichnen. Diese Bereitschaft erhöht die Akzeptanz von FDM bei den Forschenden und wird langfristig den Zugang zu Forschungsdaten erleichtern. Das Potenzial eines professionellen FDM für Forschung, Wirtschaft und Gesellschaft wurde identifiziert und eine globale Bewegung Richtung Open Science \& Data Sharing ist zu erkennen. Diese Bewegung spiegelt sich u. a. in der geplanten Implementierung nationaler und europäischer Forschungsdateninfrastrukturen (wie z.B. der NFDI oder der EOSC) deutlich wider. Und so steht die Relevanz von FDM auch auf der Agenda von Fördereinrichtungen, dem Bundesministerium für Bildung und Forschung, der Bundes- und den Landesregierungen sowie wissenschaftlichen Institutionen. Deutschland kann punktuell auf hohe FDM-Expertisen, wie beispielsweise die DINI/nestor AG Forschungsdaten, ${ }^{64}$ den RatSWD und RfII, FDM-Kompetenzzentren an den Universitäten, Landesinitiativen, ${ }^{65}$ etablierte FDMInfrastrukturen etc. sowie auf entsprechend zahlreiche FDM-Aktivitäten blicken.

Allerdings sind die Potenziale und auch karrierefördernden Aspekte eines professionellen FDM, Data Sharing und der Open Science Bewegung bisher noch nicht bis $\mathrm{zu}$ allen Forschenden und Leitungsebenen durchgedrungen. Bei den meisten Forschenden herrscht immer noch die stabile und im Wissenschaftssystem fest verankerte Mentalität des Wettbewerbs und nicht der Kollaboration, was einer disziplinübergreifenden Zusammenarbeit und schlussendlich dem wissenschaftlichen Fortschritt im Wege steht. Forschende halten ihre Erhebungsdaten oftmals lieber unter Verschluss, damit ihre Kolleginnen und Kollegen diese nicht für eigene Veröffentlichungen und somit ihren Reputationsgewinn verwenden können. ${ }^{66}$ Die GO FAIR-Initiative möchte insbesondere mit den Aktivitäten innerhalb des Handlungsfeldes GO CHANGE dafür Sorge tragen, dass Forschende dieses Misstrauen ablegen, Daten ordnungsgemäß zitiert werden und die Vorteile von Open Science und FDM in der Wissenschaft ankommen.

Ein professionelles FDM ist längst noch kein fester Bestandteil der Forschungspraxis innerhalb wissenschaftlicher Projekte geworden. Daher wäre es wünschenswert, dass Forschende die niedrigschwelligen Angebote der GO FAIR-Initiative nutzen, sich in IN auszutauschen und die Herausforderungen des FDM gemeinsam anzugehen. Die ersten Pioniere, wie etwa der RatSWD, haben dies bereits getan. Nun müssen weitere Forschungseinrichtungen und Initiativen nachziehen, so dass

64 S. https://dini.de/ag/dininestor-ag-forschungsdaten/.

65 S. z. B. Bw2FDM (https://bwfdm.scc.kit.edu/index.php); Digital Campus Bayern (https://www. stmwk.bayern.de/studenten/digitalisierung/hochschule-digitaler-campus.html); fdm.nrw (https:// www.fdm.nrw/); HeFDI (https://www.uni-marburg.de/de/hefdi).

66 Popkin 2019, 445-446; s. a. Beitrag von Oßwald, Kap. 3.5 in diesem Praxishandbuch. 
der Kulturwandel hin zu einem verantwortungsvollen FDM gelingt und die vorhandenen Synergien sinnvoll genutzt werden.

Letztendlich gehen die Ziele der GO FAIR-Initiative aber auch über die Grenzen des wissenschaftlichen Ökosystems der Forschenden hinaus. Ein wichtiges Anliegen ist nämlich darüber hinaus, das Vertrauen der Bevölkerung in das Wissenschaftssystem zu stärken oder gar verlorenes wieder zurückzugewinnen. Hierzu ist eine Offenlegung von Forschungsprozessen im größtmöglichen Umfang notwendig. Eine solche Transparenz entsteht, wenn nicht nur Forschungsergebnisse, sondern auch die dazugehörigen Forschungsdaten, Analyse-Syntaxen, Methodenreports etc. zur Verfügung gestellt werden. Erst ab dem Moment, in dem Forschungsergebnisse repliziert und überprüft werden können, handelt es sich streng genommen um Wissenschaft. Open Science erleichtert in diesem Sinne die Qualitätssicherung innerhalb der Forschung und erhöht die Leistungsfähigkeit der Wissenschaft, wovon alle Beteiligten profitieren.

Die praktische Anwendung von Open Science und die daraus folgende Transparenz fördert gesellschaftliches Vertrauen in die Wissenschaft und erhält auf Dauer ihre Glaubwürdigkeit. Schlussendlich rechtfertigt Open Science sogar ihren Fortbestand.

\section{Literatur}

Letztes Abrufdatum der Internet-Dokumente ist der 15.11.2020.

Ayris, Paul, Jean-Yves Berthou, Rachel Bruce, Stefanie Lindstaedt, Anna Monreale, Barend Mons et al. 2016. Realising the European Open Science Cloud. European Union. doi:10.2777/940154.

Brenger, Bela, Beate Baurmann, Ania López, Stephanie Rehwald und Konstantin Wilms. 2017. „Wo sind deine Forschungsdaten in 10 Jahren?!“ Awareness Für Forschungsdatenspeicherung. doi:10.5281/zenodo.1000538.

Bug, Mathias, Stefan Liebig, Claudia Oellers und Regina T. Riphahn. 2018. „Operative und strategische Elemente einer leistungsfähigen Forschungsdateninfrastruktur in den Sozial- und Wirtschaftswissenschaften.“ Jahrbücher für Nationalökonomie und Statistik 238(6): 571-590. doi:10.1515/jbnst-2018-0029.

DFG - Deutsche Forschungsgemeinschaft. 2019. „Leitlinien zur Sicherung guter wissenschaftlicher Praxis.“ https://www.dfg.de/download/pdf/foerderung/rechtliche_rahmenbedingungen/ gute_wissenschaftliche_praxis/kodex_gwp.pdf.

DFG - Deutsche Forschungsgemeinschaft. 2019. „Guidance Notes on Funding Criteria National Research Data Infrastructure (NFDI).“ https://www.dfg.de/formulare/nfdi120/nfdi120_en.pdf.

Drefs, Ines, Monika Linne und Klaus Tochtermann. 2018. FAIRe Forschung. Wie Wissenschaftliche Bibliotheken den Herausforderungen von Open Science begegnen. BuB - Forum Bibliothek und Information 70 (11): 636-639.

Droß, Patrick und Julian Naujoks. 2019. Die Kuratierung sozialwissenschaftlicher Forschungsdaten Praxisfragen und Beispiellösungen. In Forschungsdaten - sammeln, sichern, strukturieren. 8. Konferenz der Zentralbibliothek, Forschungszentrum Jülich, WissKom 2019, Jülich, Germany, 
4-6 Juni 2019, hg. v. Bernhard Mittermaier, 23-38. Handle: http://hdl.handle.net/10419/ 201387.

EOSC Declaration. 2017. „European Open Science Cloud. New Research \& Innovation Opportunities.“ https://ec.europa.eu/research/openscience/pdf/eosc_declaration.pdf\#view=fit\&pagemode $=$ none.

EOSCsecretariat. 2019. „FAIR Working Group.“ https://www.eoscsecretariat.eu/working-groups/ fair-working-group (05.11.2019).

Glaeser, Edward L., David I. Laibson, José A. Scheinkman und Christine L. Soutter. 2000. „Measuring Trust.“ Q. J. Econ. 115 (3): 811-846.

Huschka, Denis, Claudia Oellers, Notburga Ott und Gert Wagner. 2011. „Datenmanagement und Data Sharing: Erfahrungen in den Sozial- und Wirtschaftswissenschaften.“ In: Handbuch Forschungsdatenmanagement, hg. v. Stephan Büttner, Hans-Christoph Hobohm, Lars Müller, 3548. Bad Honnef: Bock+Herchen.

Linne, Monika. 2013. „Sustainable data preservation using datorium: facilitating the scientific ideal of data sharing in the social sciences." Proceedings of the 10th International Conference on Preservation of Digital Objects: 150-155.

Linne, Monika und Wolfgang Zenk-Möltgen. 2017. „Strengthening institutional data management and promoting data sharing in the social and economic sciences.“ LIBER Quarterly 27 (1): 58 72. doi:10.18352/lq.10195.

Open Science Monitor. 2019. https://ec.europa.eu/info/research-and-innovation/strategy/goalsresearch-and-innovation-policy/open-science/open-science-monitor/facts-and-figures-openresearch-data_en.

Popkin, Gabriel. 2019. Data sharing and how it can benefit your scientific career. Nature 569 (7756): 445-447. doi:10.1038/d41586-019-01506-x.

Rehwald, Stephanie und Bela Brenger. 2019. „Projekt UNEKE: Von USB-Sticks und Repositorien Ergebnisse der UNEKE-Umfrage. DINI/nestor-Workshop. 29.01.2019. Duisburg-Essen.“ https:// www.forschungsdaten.org/index.php/Datei:01-uneke-usb-sticks-und-repositorien.pdf.

RatSWD 2019a: Remote Access zu Daten der amtlichen Statistik und der Sozialversicherungsträger. RatSWD Output 5 (6). Berlin: Rat für Sozial- und Wirtschaftsdaten (RatSWD). doi:10.17620/ 02671.42 .

RatSWD. 2019b. Tätigkeitsbericht 2018 der vom RatSWD akkreditierten Forschungsdatenzentren (FDZ). doi:10.17620/02671.38.

RfII - Rat für Informationsinfrastrukturen. 2016. „Leistung aus Vielfalt. Empfehlungen zu Strukturen, Prozessen und Finanzierung des Forschungsdatenmanagements in Deutschland.“ http:// www.rfii.de/?p=1998.

Royal Society. 2012. „Science as an open enterprise. Royal Society Science Policy Centre Report 02/12." https://royalsociety.org/policy/projects/science-public-enterprise/Report/.

Vocile, Bobby. 2017. „Open Science Trends You Need to Know About.“ https://www.wiley.com/network/researchers/licensing-and-open-access/open-science-trends-you-need-to-know-about.

Wilkinson, Mark, Michel Dumontier, IJsbrand Jan Aalbersberg, Gabrielle Appleton, Myles Axton, Arie Baak et al. 2016. „The FAIR Guiding Principles for scientific data management and stewardship." Scientific Data 3: doi:10.1038/sdata.2016.18.

Zenk-Möltgen, Wolfgang, Esra Akdeniz, Alexia Katsanidou, Verena Naßhoven und Ebru Balaban. 2018. „Factors influencing the data sharing behavior of researchers in sociology and political science." Journal of Documentation 74 (5): 1053-1073.doi:10.1108/JD-09-2017-0126.

ZBW. 2019. „Die GO FAIR Initiative.“ https://www.zbw.eu/de/ueber-uns/arbeitsschwerpunkte/forschungsdatenmanagement/go-fair/. 